\title{
Cognitive Behavioral Therapy for Tinnitus Distress
}

\author{
Melehin AI* \\ Cognitive Behavioral Psychotherapist, Russia
}

*Corresponding author: Melehin AI, PhD, associate Professor, clinical psychologist of the highest qualification category, cognitive behavioral psychotherapist. Stolypin Humanitarian Institute, Moscow, Russia.
Received Date: June 05, 2020

Published Date: June 22, 2020

\begin{abstract}
s
In the article, tinnitus is considered as a biopsychosocial disorder that leads patients to tinnitus-specific distress. Approaches and psychotherapeutic targets for reducing tinnitus distress are systematized. The protocol of clinical and psychological examination of a patient with noise/ringing in the ears, which allows you to identify the targets of therapy, track the dynamics of treatment and minimize barriers, is presented. The specifics and effectiveness of the protocols of the «first», «second» and third waves of cognitive-behavioral psychotherapy of tinnitus.

Key words: Tinnitus; Cognitive behavioral psychotherapy; Tinnitus distress
\end{abstract}

\section{Mini Review}

Over the past few years, patients with complaints of noise/ ringing in the ears have been increasingly seeking psychological advice in Russia. The patients themselves describe sounds as «buzzing», «squeaking», «clock ticking», «hissing», «roaring». Most often, these patients turn to a clinical psychologist on the recommendation of a doctor-an otorhinolaryngologist, audiologist, or neurologist. The presence of such noise in the ears is called tinnitus (subjective tinnitus) - a subjective («phantom») auditory perception (not an auditory hallucination), which is the result solely of the activity of the nervous system, without any participation of mechanical, vibrational effects on the inner ear [1-6]. This disorder is common in $10-15 \%$ of the adult population, and due to its multifactor origin, it is accompanied by a heterogeneous course profile in each patient. For some people, the presence of tinnitus, although constantly present, is perceived as harmless, while for others it is accompanied by a strong tinnitus-specific distress (tinnitus-related distress) in the form of [4]:

- $\quad$ impaired concentration;
- insomnia;

- $\quad$ careful monitoring of sounds in the ears;

- a disastrous style of thinking;

- $\quad$ anxious ruminations;

- a spectrum of avoidant and problem-oriented behavior.

Up to $70-80 \%$ of patients suffering from tinnitus have mental comorbidities (depression, anxiety spectrum disorders) that affect the quality of life. The presence of tinnitus is inextricably linked to the patient's attention disorders, which affect the process of habituation and do not inhibit the "phantom" auditory perception [2].

Similar to chronic pain, tinnitus can be viewed as a distorted perception of illness/harm, rather than as a «true» illness. The presence of disturbing tinnitus is a negative emotional and auditory experience that is perceived by the patient as carrying actual or potential physical or psychological harm. To this day, this disorder 
is not treated, there is no effective pharmacological, instrumental tactics. The use of psychopharmacotherapy does not directly affect tinnitus, causing a range of additional side effects in patients. For example, dizziness, the appearance of «new noises» $[2,6]$.

To this day, both in Russia and foreign otorhinolaryngological and neurological practice, there is no standardized treatment tactics, so we are talking more about the tactics of cognitive behavioral management of symptoms due to the fact that tinnitus is mainly explained more by the psychological characteristics of the patient. For example, reactions to fear, cognitive distorted interpretations of bodily sensations, negative emotional reactivity, selective attention processes, and avoiding behavioral strategies [6]. Changes in cognitive control and a shift in attention negatively directed at oneself affect tinnitus [3].

The role of «psychosomatic» and stress responses in the development of tinnitus is confirmed by a number of modern pathogenetic models. For example, the neurophysiological model proposed by Jastreboff. (neurophysiological approach to tinnitus), according to which tinnitus becomes chronic and decompensated due to impaired functioning of the circuit in a complex neural network that includes sensory, limbic and vegetative components. There is a connection between the subjective perception of tinnitus

Table 1: Approaches and psychotherapeutic targets for reducing tinnitus distress

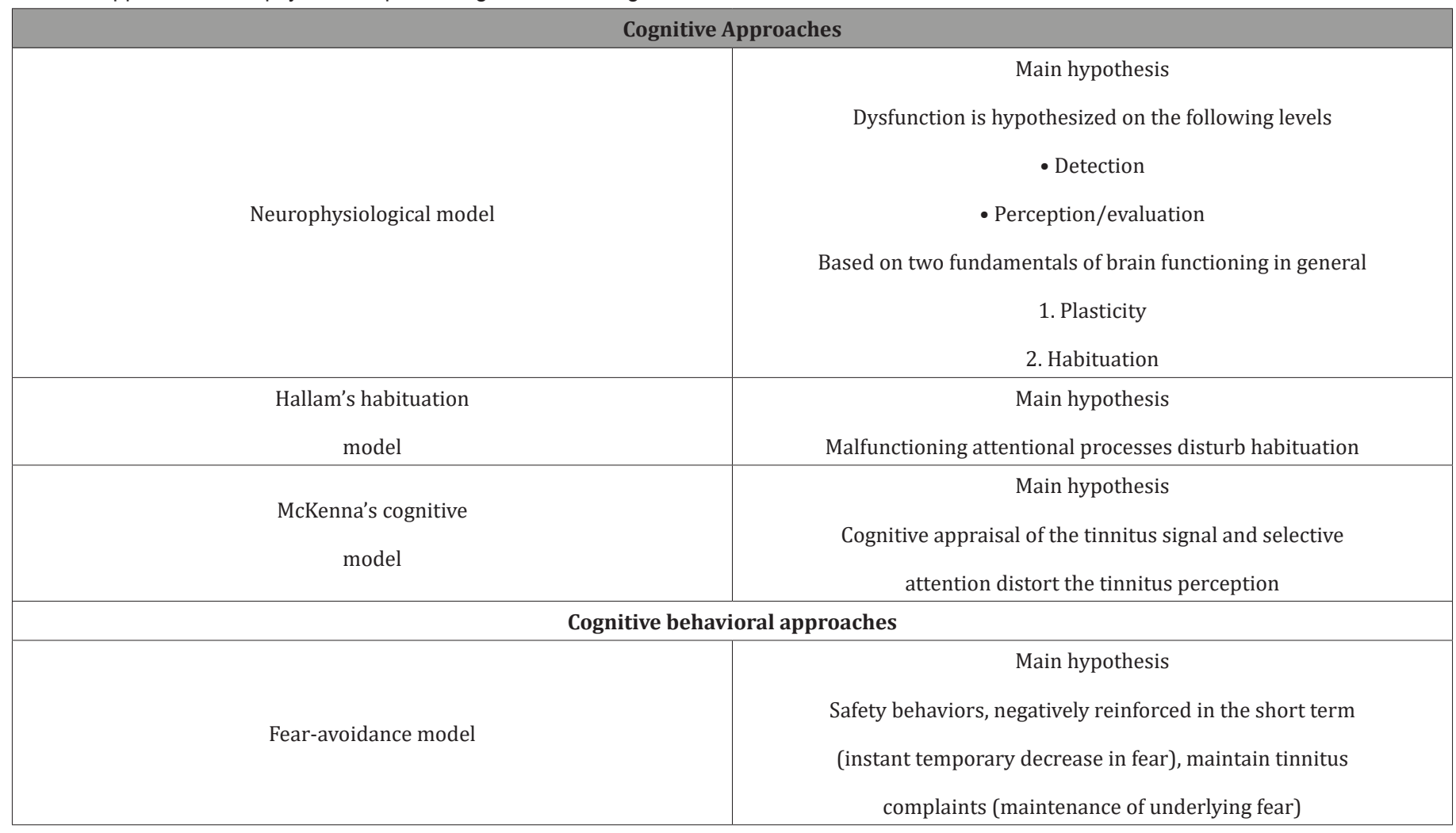

We distinguish the following tactical lines of cognitivebehavioral psychotherapy of tinnitus (CBT-T):

1) Demystification of tinnitus and ensuring maximum understanding of the patient's own condition in order to and excessive spontaneous electrical / metabolic activity in the auditory center, limbicsystem, and changes in the autonomic nervous system. Thus, most often the basis of tinnitus is dysregulation in the nervous system, which can be caused by various psychological reasons. Since the updated Cochrane review of psychotherapeutic approaches to the treatment of tinnitus in 2020 was published [4], cognitive behavioral therapy for tinnitus (CBT-T) is considered the «gold standard» of treatment. The multi-disciplinary European guidelines for the treatment of tinnitus 2019 [2] considers CPR as the «first line». It can be used with pharmacotherapy (ginkgo biloba, BzD), audiological means (hearing AIDS, cochlear implants), TMS.

CBT-T is based on the following models: the habituation model of Hallam (habituation model tinnitus, R. Hallam); the neurophysiological model of tinnitus P. Jastreboff (neurophysiological model tinnitus, P. J. Jastreboff), and the cognitive behavioral model of tinnitus L. Makenna (conceptual cognitive model tinnitus, McKenna) [2,6].

In Table 1, we show that the existing models of tinnitus underlying the psychological approach can be divided into two areas with counseling psychotherapeutic targets (Table 1). 
character. Restoring the threshold of auditory experiences is accomplished by masking - using any sound that provides some immediate relief for tinnitus. Also, the selection of therapeutic sound or emotional «anchor» - soothing sounds, smells, thrills. Gabbitation also includes techniques for managing attention, translating it from a scanning, problem-oriented position;

3) Reducing maladaptive responses (both cognitive and behavioral) using «de-catastrophization» techniques and exposure algorithms.

At the beginning, middle and end of CBT-T, we recommend conducting a clinical and psychological examination of a patient with tinnitus according to our proposed diagnostic protocol (table.2) to assess the dynamics of psychotherapy treatment and minimize barriers (for example, «why did I try everything but nothing worked? am I special, a loser?») (Table 2).

Table 2: Clinical and psychological examination of a patient with tinnitus.

\begin{tabular}{|c|c|}
\hline $\begin{array}{l}\text { Distress/Severity: General measures of severity which are mostly hybrid, since } \\
\text { they are intended to capture the multifaceted nature of tinnitus distress }\end{array}$ & $\begin{array}{l}\text { - THI Tinnitus Handicap Inventory } \\
\text { - } \quad \text { TQ Tinnitus Questionnaire } \\
\text { - TRQ Tinnitus Reaction Questionnaire } \\
\text { - TSI Tinnitus Severity Index } \\
\text { - THQ Tinnitus Handicap Questionnaire } \\
\text { - TSQ Tinnitus Severity Questionnaire } \\
\text { - TFI Tinnitus Functional Index }\end{array}$ \\
\hline $\begin{array}{l}\text { Psychological mechanisms: instruments designed to assess the specific psy- } \\
\text { chological } \\
\text { domains which are considered predictive for tinnitus distress/severity }\end{array}$ & $\begin{array}{c}\text { - TCS Tinnitus Catastrophizing Scale } \\
\text { - } \quad \text { FTQ Fear of Tinnitus Questionnaire } \\
\text { - TVAQ Tinnitus Vigilance and Awareness Questionnaire } \\
\text { PVAQ Pain Vigilance and Awareness Questionnaire } \\
\text { - } \quad \text { T-FAS Tinnitus Fear-Avoidance Scale } \\
\text { - TAQ Tinnitus Acceptance Questionnaire } \\
\text { - TDI Tinnitus Disability Index }\end{array}$ \\
\hline
\end{tabular}

At the moment, there are the following protocols of the checkpoint, which are conducted mainly in a face-to-face and individual format:

I. Protocols CBT of the «first» wave:

- Tinnitus Retraining Therapy includes: 1) change the interpretation (negative thoughts, beliefs about tinnitus) using independent step-by-step instructions; 2) sound therapy with the use of disguise, avoid silence by enriching the environment with sounds. This leads to the habituation of auditory sensations. The effectiveness of this Protocol remains unconvincing and ineffective to this day [6].

II. Protocols CBT of the «second» wave:

- Exposure therapy for tinnitus is aimed at reducing the perception of a neutral signal (harmless tinnitus) and an aversive interoceptive stimulus (sympathetic arousal), which allows you to remove the fixed fear response, i.e. stable conditional reactions (negative emotional response, excessive monitoring, increased awareness, the spectrum of avoiding behavior). This is more effective than imposing a short-term habituation to the stimulus through disguises on the patient. The emphasis is on the opposite: confrontation instead of avoidance, exposure instead of disguise, awareness of tinnitus instead of distraction, observation of thoughts instead of changes. The emphasis is on reducing excessive «cognitive armor» in the form of catastrophization («I will live with this all my life», «because of the very fact of noise, I can not completely relax, live a happy life») and maladaptive schemes (strict internal standards; negativity/pessimism in the perception of information; «vulnerability» to something. For example, diseases). Remission of 12 months in which there is a decrease in tinnitus, catastrophization of tinnitus, and symptoms of anxiety $[1,4]$.

III. «Third» wave CBT protocols:

- Mindfulness-based stress reduction therapy, which is aimed at developing the patient's skills of broad and flexible attention, instant awareness and observation of emotions, feelings, and thoughts without evaluation. The principle of mindfulness is that if the patient stops trying to avoid unpleasant sensations (such as tinnitus), he will be able to perceive it without reactions of panic and struggle. This approach offers patients a new way to relate to their own thoughts, which will allow them to be able to manage their propensity for negative and disastrous thoughts. Classes are 
built around meditative skills ("body scan without evaluation", "three-minute meditation", conscious actions), physical exercise, and psychoeducation. The average number of sessions is 6-7. Remission of 6 months [5].

- Acceptance and commitment therapy focuses on the functional utility of thoughts and actions, rather than their «rightness and illegality». One of the key elements of action is to help the patient flexibly evaluate psychological events (thoughts, perception of symptoms, emotions) without judging, without trying to change or modify them. This leads to a flexible functional awareness that thoughts, emotions, and behavior create and maintain distress and tinnitus. Since the skills of broad attention contribute to awareness and observation of the present moment without judgment, they have become an integral part of this therapy [7].

- Metacognitive therapy focuses on metacognitive beliefs, experiences, and strategies (the ability to observe your thinking «as if from above», not fight it or change it). The presence of distorted strategies can lead to various patterns of dysfunctional thoughts and disturbing ruminations. Treatment begins with the «separation» tactic (attention training technique), which involves stopping or disabling any response to a thought or behavior. Learn to experience yourself as separate from ideas and behavior. Next, we learn to increase awareness (detached mindfulness) focused on the ability to distinguish negative thoughts from the subsequent maladaptive response to this thought. 8 sessions, 60 minutes each two weeks. Remission of 3 months consisted in the absence of symptoms of depression, anxiety, catastrophization of tinnitus [3].

\section{Conclusion}

Thus, CBT-T is a potentially effective tactic for reducing tinnitus distress, symptoms of depression and anxiety in patients with tinnitus.

\section{Acknowledgement}

None.

\section{Conflict of Interest}

No conflict of interest.

\section{References}

1. Cima RF, Maes IH, Joore MA (2012) Specialised treatment based on cognitive behaviour therapy versus usual care for tinnitus: a randomised controlled trial. Lancet 37(3): 1951-1959.

2. Cima RF, Mazurek B, Haider H, D Kikidis, A Lapira, et al. (2019) A multidisciplinary European guideline for tinnitus: diagnostics, assessment, and treatment. HNO 67: 10-42.

3. Ferraro L, La Barbera D, Aldo M, Simona G, Anna M, et al. (2019) Metacognitive Therapy in Patients with Tinnitus: a Single Group Study. Journal of Contemporary Psychotherapy 49: 133-140.

4. Fuller T, Cima R, Langguth B, Birgit M, Angus W, et al. (2020) Cognitive behavioural therapy for tinnitus (Protocol). Cochrane Database Syst Rev: 110.

5. McKenna L, Marks EM, Hallsworth CA (2017) Mindfulness-based cognitive therapy as a treatment for chronic tinnitus: a randomized controlled trial. Psychother Psychosom 86(6): 351-361.

6. Textbook of Tinnitus. / ed Aage R. Møller, Berthold Langguth, Dirk De Ridder. NY. Springer, 2011, 762

7. Westin VZ, Schulin M, Hesser H, Karlsson M, Noe RZ, et al. (2011) Acceptance and commitment therapy versus tinnitus retraining therapy in the treatment of tinnitus: a randomised controlled trial. Behav Res Ther 49(11): 737-747. 